\title{
LV-SY-2-4
}

\section{Validation of Hong Kong liver cancer staging system in resectable HCC}

\author{
Tan To CHEUNG*
}

Department of Surgery, The University of Hong Kong, Hong Kong, China

Lecture: In Hong Kong, liver cancer is the fifth most common cancer and the third most common cause of cancer deaths. The prevalence of hepatitis B is high in Hong Kong because of the high rate of hepatitis B virus infection, and chronic hepatitis B has remained the leading cause of hepatocellular carcinoma in the city, accounting for $80 \%$ of all cases in the period from 1992 to 2016 . In view of the different etiologies of hepatocellular carcinoma around the world, a group of liver experts in Hong Kong developed the Hong Kong Liver Cancer staging system in order to provide more aggressive treatment guidance (predominantly a wider use of surgical resection) for Asian patients of hepatocellular carcinoma. In this article focussing on the Hong Kong Liver Cancer staging system, we briefly reviewed the screening criteria adopted in Hong Kong for liver resection, local ablation, transcatheter arterial chemoembolization, transcatheter arterial radioembolization, stereotactic body radiation therapy, and systemic therapy. 